This is the final peer-reviewed accepted manuscript of:

THE DESIGN OF MINI/MICRO HEAT EXCHANGERS: A WORLD OF OPPORTUNITIES AND CONSTRAINTS

in Proceedings of the 16th International Heat Transfer Conference, IHTC-16. Danbury, CT (USA); Beijing (PRC), Begell House, 2018. pp. 469-486.

The final published version is available online at:

http://dx.doi.org/10.1615/ihtc16.kn.000028

Rights / License:

The terms and conditions for the reuse of this version of the manuscript are specified in the publishing policy. For all terms of use and more information see the publisher's website.

This item was downloaded from IRIS Università di Bologna (https://cris.unibo.it/)

When citing, please refer to the published version. 


\title{
THE DESIGN OF MINI/MICRO HEAT EXCHANGERS: A WORLD OF OPPORTUNITIES AND CONSTRAINTS
}

\author{
Gian Luca Morini ${ }^{1}$, Juergen J. Brandner ${ }^{2}$ \\ ${ }^{1}$ Alma Mater Studiorum Università di Bologna, Viale Risorgimento 2, 40136 Bologna, Italy \\ ${ }^{2}$ IMT, Karlsruhe Institute of Technology, Institute of Microstructure Technology (IMT), Hermann-von- \\ Helmholtz-Platz 1, 76344 Eggenstein-Leopoldshafen, Germany
}

\begin{abstract}
Micro heat exchangers and heat sinks broadened their use in many technological fields during the last two decades. The reduction of the dimensions of the channels allows to obtain ultra-compact heat exchangers characterized by higher surface-to-volume ratio and overall heat transfer coefficients but, in general, with large pressure losses. Many imaginative configurations have been proposed and tested, by changing the geometry of the manifolds, the position of the inlet/outlet ports, the structure of the heat transfer core, the structural materials and others more. Unfortunately, these efforts were not coordinated and a complete overview of the results accumulated up to now is not available. However, some general conclusions can be made by using the published results and the main scope of this paper is to summarize these milestones. Some shared conclusion are the following: (i) the design of micro heat exchangers can be obtained by using the classical methods developed for conventional heat exchangers even if the presence of non- negligible scaling effects (i.e. compressibility effects, conjugate wall-fluid effects, viscous dissipation) must be always verified; (ii) the performances of micro heat exchangers and heat sinks is strongly influenced by the proper distribution of the flow rate within the heat transfer core and a series of different solutions is available in order to solve this problem, as summarized in this paper; (iii) the presence of strong conjugate wall-fluid heat transfer effects can become an opportunity for the use of miniaturized heat exchangers made with inexpensive materials having low thermal conductivity values, especially in presence of counter-current flow and cross-flow configurations.
\end{abstract}

KEY WORDS: Microchannels, Heat Exchangers, Manifolds, Pressure losses, Overall heat transfer coefficients, Flow configuration, Thermal effectiveness.

\section{INTRODUCTION}

In the last twenty years, many conventional devices in which heat transfer and/or chemical reactions are performed were scaled down from macro-metric sizes to micrometric dimensions in order to improve their performances together with their portability [1-3]. Among these micro-structured devices, micro heat exchangers (mHEX) and micro heat sinks (mHS) are of increasing interest for their improved heat exchange capacities due to very large surface-to-volume ratio and very short heat transfer time.

An extended review of the main researches on micro heat exchangers was made by Dixit and Ghosh [4] for single phase fluids. More recently, Ahmed et al. [5] published a critical overview of the results obtained during the last twenty years about the optimization of the thermal design of micro heat sinks. Some conclusions can be summarized by these two reviews: (i) a big effort has been made during the last decades in order to verify if the scaling laws are valid in order to study the behavior of miniaturized heat sinks and heat exchangers; (ii) fabrication techniques have improved the capability to make reproducible microgeometries for the heat transfer surfaces but they are still expensive if dimensional inaccuracy and bonding problems have to be avoided; (iii) many materials can be now used for the fabrication of micro heat exchangers and micro heat sinks, including nano-porous media and metallic foams. 
From a review of the studies appeared in literature it is evident how the applied research on mHEX using liquids (mostly deionized water) as working media can be considered extensive [6-9], even if many conflicting results (compared with the prediction of the conventional theory) exists there and in many cases these differences have not been fully clarified up to now. On the contrary, the experimental investigation of mHEX in which gas flows as working fluids are used, is fragmented and incomplete until now [10-14]. It is important to mention that many patents have been submitted in the last two decades in this field and this fact has reduced the open access to many experimental results obtained within the academic and industrial Labs. As an example, a series of interesting applications of mHEX and mHS has been developed for data center cooling but many of these applications are covered by industrial patents (i.e. Aquatron ETH-IBM project [15]).

However, the reduction of the inner dimensions of the channels implies numberous negative effects which cannot be ignored during the design of a new micro heat exchanger. In fact, very small channels means, among others: (i) manufacturing problems, complex bonding and fitting management; (ii) large pressure losses and (iii) accentuated fouling problems. Usually, the optimal design of a micro heat exchanger is obtained as a trade-off among the desired thermal performances, the acceptable pressure losses and other specific constraints (i.e. fouling, costs, mechanical resistance and so on). Some of these constraints can be satisfied with a targeted design. As an example, it is possible to limit the value of the maximum pressure loss across the mHEX or mHS by subdividing the active surface of these devices in parallel microchannels thanks to two manifolds at the inlet and at the outlet. In many cases, the adopted number of parallel microchannels is large (>30) and this aspect introduces a new problem related to the presence of a mal-distribution of the working fluid among the channels. Flow mal-distribution can be very critical especially in evaporators and condensers: in presence of two-phase flows this can trigger hydraulic instabilities. How the non-uniform distribution among the channels can be controlled is explained in this paper by summarizing the main solutions proposed to overcome this problem.

As indicated in many papers $[1,3,4]$, the design of micro heat exchangers in many cases can be based on conventional methods and correlations developed for classical heat exchangers; however, as demonstrated by Morini and Yang [16], the presence of non- negligible scaling effects (i.e. compressibility effects, conjugate wall-fluid effects, viscous dissipation) must be always verified before to use conventional correlations for the design of mHEX and mHS. In fact, in presence of significant scaling effects the behavior of mHEX and mHS can deviate from the expected conventional behavior and these deviations can represent, in some case, an opportunity in order to obtain unique performances from mHEX and mHS.

As an example, a special feature of mHEX and mHS is that thickness of the solid walls can be of the same order of magnitude of the hydraulic diameters of the channels; under these conditions the conjugate heat transfer between the solid walls and the fluids cannot be ignored both along the axial and transversal direction of the device. The works on mHEX with gas flows have highlighted that the role of wall axial conduction is never negligible in presence of thick solid walls; in this case the thermal performance of miniaturized gas heat exchangers becomes quite independent by the flow arrangements (cross flow, cocurrent flow and counter-current flow) as well as by the wall material. Many experimental results $[13,14]$ suggest that, in order to obtain larger heat transfer rates, material of lower thermal conductivity (compared with metal) should be used both for microchannel plates and for the shell of micro heat exchangers, as long as the overall heat transfer coefficient is not significantly reduced by the increase in the thermal resistance between the hot and cold flows. This result is very interesting because it demonstrates that two beneficial effects can be obtained from a peculiar aspect of mHEX and mHS like the non -negligible presence of conjugate wall-fluid heat transfer effects: (i) in combination with gaseous flows, materials like polymers can be used for the fabrication of miniaturized gas heat exchangers without to obtain strong penalties in terms of heat transfer efficiency; (ii) the use of plastic materials enlarges the microfabrication techniques available for the production of mHEX and mHS to, in example, 3D printers with a significant reduction of the costs for a customized production of mHEX and mHS for specific applications.

The interest of the heat exchanger industry to the introduction of polymeric materials for the massive production of heat exchangers has been critically analyzed by Chen et al. [17] in 2016; the authors concluded that polymer heat exchangers can potentially replace the conventional metallic heat exchangers in a wide range of applications but a considerable amount of research is still required before to consider these replacement as efficient. In this paper it will be demonstrated that polymers can be proficiently used in $\mathrm{mHEX}$ in presence of gaseous working fluids. 
Since the topic is very large, this paper will focus the attention on two aspects which are important for the optimization of the mHEX and mHS:

- The role of the inlet and outlet manifolds for the reduction of the pressure loss and of the flow maldistribution problems.

- The role of conjugate heat transfer between solid walls and fluids on the heat exchanger efficiency.

The examples shown in this paper clarify that the miniaturization of the channels of a heat exchanger is, for sure, a source of problems (i.e. large pressure loss, flow mal-distribution and so on) but with an oriented design these problems can be transformed, in some cases, in useful opportunities.

\section{THE DESIGN OF MICRO HEAT EXCHANGERS AND HEAT SINKS}

The author's experience accumulated during the last two decades on the experimental and numerical analysis of mHEX suggests that the conventional theory can still be used for the prediction of both pressure losses and overall heat transfer coefficients (and hence for the design) of mHS and mHEX with channels having characteristic dimensions in the range of some ten to some hundred $\mu \mathrm{m}[1,16]$. The $\varepsilon$-NTU method or the logarithmic mean temperature difference method are applicable for the design of these devices and the classical theory, developed for conventional heat exchangers, can be applied to the reduction of the experimental data. The heat transfer rate can be calculated by measuring the temperature difference between the inlet and the outlet and the mass flow rate on the hot side:

$$
\dot{Q}_{h / c}=q_{t o t, h / c} c_{p, h / c}\left(T_{h / c, \text { in }}-T_{h / c, \text { out }}\right)
$$

where the subscripts $h / c$ refers to the hot and cold side, $\mathrm{q}_{\mathrm{tot}}$ is the mass flow rate and $c_{p}$ is the specific heat capacity of the fluid.

The log-mean temperature difference (LMTD) of a counter-current heat exchanger is defined by:

$$
\Delta T_{L M T D}=\frac{\left(T_{h, \text { out }}-T_{c, \text { in }}\right)-\left(T_{h, \text { in }}-T_{c, \text { out }}\right)}{\ln \frac{T_{h . \text { out }}-T_{c, \text { in }}}{T_{h, \text { in }}-T_{c, \text { out }}}}
$$

Ideally the heat transfer rate obtained from both sides should be equal. However, in practice the values of these two parameters may slightly differ from each other. Therefore, in data reduction the heat transfer rate is usually averaged from both sides:

$$
\dot{Q}_{a v e}=\frac{\dot{Q}_{h}+\dot{Q}_{c}}{2}
$$

The overall heat transfer coefficient $k$ is calculated by:

$$
k=\frac{\dot{Q}_{a v e}}{F A \Delta T_{L M T D}}
$$

where $A$ is the heat transfer area and $F$ is a correction factor which depends on the flow configuration ( $F=1$ for countercurrent and parallel flow configurations).

The heat exchanger effectiveness, defined by the ratio of the real heat transfer rate and the maximum capacity of heat transfer rate, is given by:

$$
\varepsilon=\frac{\dot{Q}_{a v e}}{\left(q_{\text {tot }} c_{p}\right)_{\min }\left(T_{h, \text { in }}-T_{c, \text { in }}\right)}
$$

The number of transfer unit (NTU) is defined as usual:

$$
N T U=\frac{k A}{\left(q_{t o t} c_{p}\right)_{\min }}
$$


For the evaluation of pressure losses and overall heat transfer coefficients the conventional correlations (i.e. Gnielinski correlation [18]) can be generally employed even if in literature contradictory results about this point can be found.

The author's experience highlights that deviations from the conventional correlations are possible only in presence of effects which assume a strong importance when the channel dimensions are reduced (scaling effects). For this reason, as underlined in [16], during the design of a new mHS or mHEX an "a priori" evaluation of the weight of each scaling effect becomes mandatory. Morini and Yang [16] reported a list of the main scaling effects which can be encountered in mHEX and mHS and they associated a threshold value of a dimensionless quantity to each scaling effect in order to drive their "a priori" evaluation. The most important scaling effects which can influence the performances of mHS and mHEX are:

- Conjugate solid-wall heat transfer (especially in presence of gas flows): this effect is originated by the large thickness of the partition walls among the microchannels which is of the same order of magnitude of the inner dimensions of the microchannels. The presence of a strong conjugate heat transfer tends to reduce the value of the heat exchanger effectiveness and this reduction is stronger for larger NTU values, which means in presence of larger overall heat transfer coefficients and lower mass flow rates (this is exactly the case of mHEX and mHS)

- Viscous dissipation (for high velocity of both liquid and gas flows): the working fluids tend to be heated along the channels because they are forced to flow in small passages. This heating tends to reduce the values of the overall heat transfer coefficients when the Reynolds number is increased.

- Compressibility effects (for high velocity gas flows): in presence of gas flows the velocity reached by the fluid within the micro-channels can be very high even if the flow is laminar regime. The effect due to the variation of the gas density along the microchannels (due to the pressure decrease along the channels) influences the values of both friction factors (which become a function of the Mach number) and heat transfer coefficients (introducing local cooling effects linked to the gas expansion). For very large mass flow rates (and gas velocities) chocked flow conditions can be reached along the mHEX.

All these effects can be correctly modelled during the design of a mHEX or mHS by using conventional CFD codes [16].

\section{THE LAY-OUT OF MICRO HEAT EXCHANGERS AND HEAT SINKS}

It is well known that a reduction of the inner diameter of a channel is responsible of an increase of the convective heat transfer in laminar regime; in fact, since the Nusselt number can be considered as a constant in the fully developed region (in laminar regime), the convective heat transfer coefficient can be considered as inversely proportional to the inner diameter of the channel. On the other hand, a reduction of the channel dimension for a fixed value of the mass flow rate is responsible of an increase of the pressure losses, which scales with the inverse of the fourth power of the inner diameter, following the Darcy-Weisbach equation. This observation highlights that the miniaturization of a channel is usually responsible of the simultaneous increase of pressure loss and heat transfer coefficient. However, the huge increase of the pressure loss can be avoided: (i) by using a series of $n$ parallel miniaturized channel by means of which it is possible to subdivide the mass flow rate among the channels (ii) by reducing the length of each channel. In this way the pressure loss can be fixed at the desired value even if the inner dimensions of the channels are reduced. In a system of $n$ parallel channels, each one of length $L$, it becomes possible to fix the value of the total pressure loss by changing the number of the channels $(n)$, their length $(L)$ and inner diameter $(d)$.

As shown by Palm [19], the product between the overall heat transfer coefficient $(k)$ and the surface area $(A)$ must be constant to transfer a fixed amount of heat under a fixed mean temperature difference between the fluids $(\Delta \mathrm{T})$. In a system in which the heat transfer surface is obtained by putting together $n$ channels having an inner diameter $d$ and length $L, k A$ is constant if $n L$ is constant. In Table 1 it is shown in which way the main quantities involved in the design of a heat exchanger based on a series of $n$ parallel channels tend to scale with the number of parallel channels, their length and inner diameter. For a fixed thermal power transferred $(n L=c o n s t)$, a reduction of the inner dimension of the channels of one order of magnitude $\left(d_{2}=d_{1} / 10\right)$ determines an increase of the heat transfer coefficient by a factor 10 , a decrease of the heat 
transfer surface of a factor 10 but with the consequent reduction of the volume by a factor 100 . This means that the surface to volume ratio $(A / V)$ of the system increases by a factor 10 , like $k$. In order to maintain the pressure loss constant, the product $\left(n d^{2}\right)$ must be constant; this means that the number of the parallel channel must be increased by a factor 100 . At the same time, the length $L$ must be reduced by a factor 100 . This simple exercise highlights that it is possible to avoid large pressure losses even if very small channels are used in a heat exchanger by increasing the number of channels in parallel.

Table 1. Scaling laws of the main quantities involved in the heat exchanger design for a configuration of $n$ parallel channel of length $L$ and inner diameter $d$.

\begin{tabular}{|c|c|c|c|c|c|}
\hline & $\mathbf{Q}_{\text {th }}$ & $\mathbf{k}$ & $\Delta \mathbf{p}$ & $\mathbf{A}$ & $\mathbf{V}$ \\
\hline \multirow{2}{*}{ Scaling law } & $L n$ & $\frac{1}{d}$ & $\frac{L}{n d^{4}}$ & Lnd & Lnd $^{2}$ \\
\hline
\end{tabular}

In order to operate with low pressure losses in mHEX and mHS a large number of parallel microchannels are typically used $(n>30)$ per single layer. One or two layers are generally used in order to obtain the heat transfer core of a mHS. On the contrary, different layers are stacked together in order to obtain the heat transfer core of mHEX.

mHEX and mHS can be always subdivided in three regions:

1) Inlet and outlet ports

2) Inlet and outlet manifolds

3) Heat transfer core

The inlet and outlet ports are the connections with the external world of mHEX and mHS; these ports introduce the working fluids: (i) orthogonally or (ii) in parallel to the direction of the fluid within the heat transfer core. A series of different configurations can be generated, by changing the positions of the ports, of the manifolds and the structure of the heat transfer core, as shown in Figure 1.

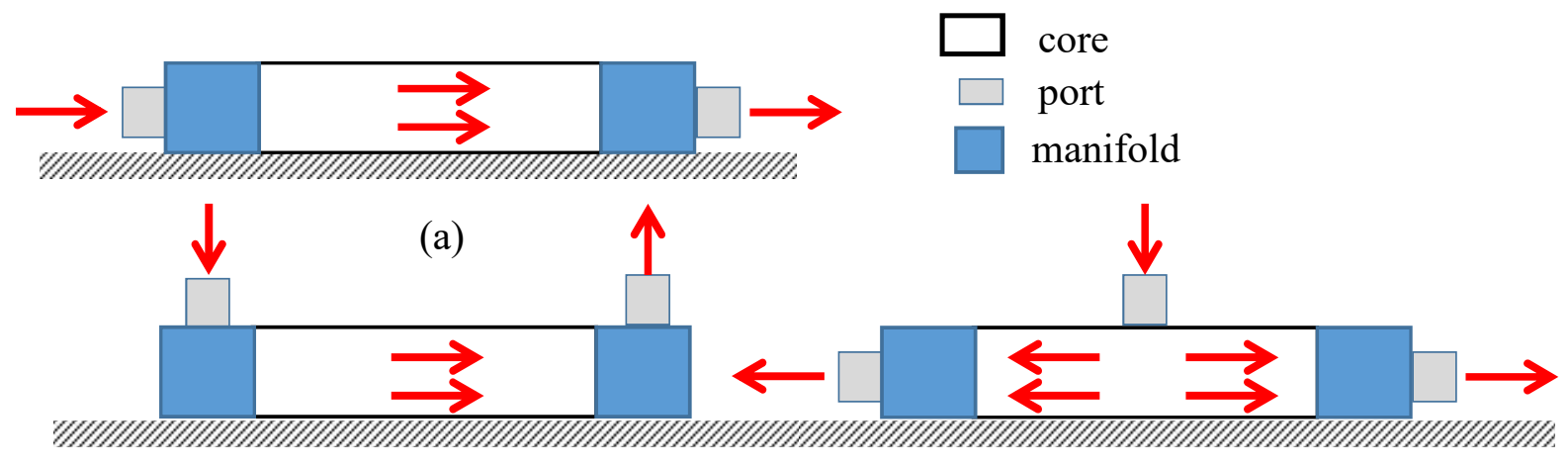

(b)

(c)

Figure 1. Typical configurations for mHS.

In Figure 1a the inlet port introduces the fluid in parallel with respect to the direction of the flow within the core of the mHS; in Figure $1 \mathrm{~b}$ the fluid is introduced orthogonally to the direction of the flow along the heat transfer core of mHS. In Figure 1c the fluid is introduced orthogonally to the core and collected peripherically by an outlet manifold. Hybrid configurations are still possible.

In mHEX, a double heat transfer core is present in which two working fluids flow (cold and hot fluid); this means that in order to obtain a mHEX a minimum number of two layers are generally stacked together; in Figure 2 the heat transfer core of a double-layered mHEX (Figure 2a) and of a multi-layered mHEX (Figure $2 b$ ) is shown; the multi-layered mHEX is generally obtained by alternating different layers in which the cold and the hot fluid flow. 


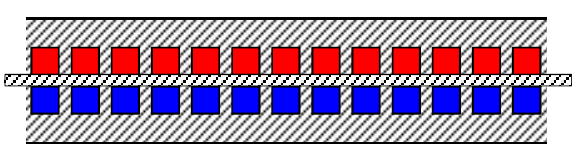

(a)

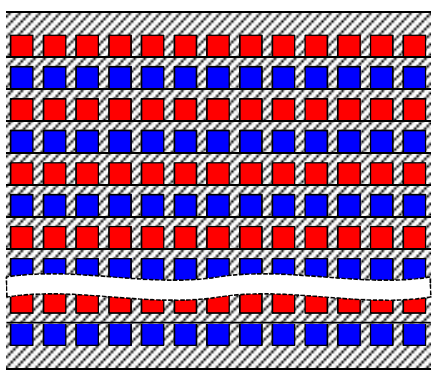

(b)

Figure 2. Schematic of (a) double-layer mHEX; (b) multi-layer mHEX.

The core of a multi-layered mHEX can allow various flow arrangements (countercurrent, co-current and cross flow) even for a fixed core structure, by changing the position of the inlets and of the manifolds, as well as the flow direction across the core. In Figure 3 a double layer mHEX is shown in which the layers can be rotated in order to obtain, with the same device, countercurrent, cross and co-current flow arrangements.

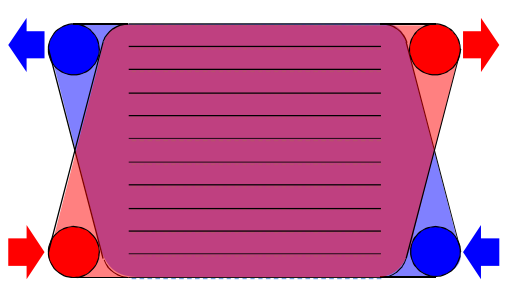

(a)

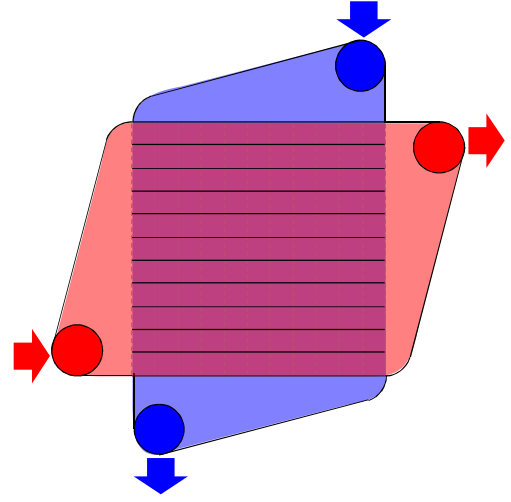

(b)

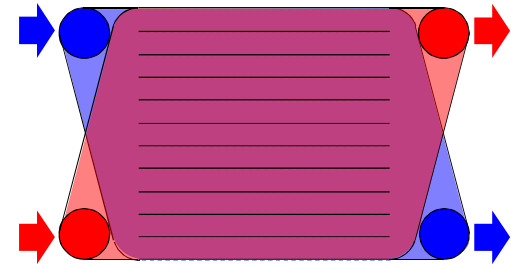

(c)

Figure 3. Three flow arrangements achievable in a double-layer mHEX [13].

Many geometries and configurations of ports, manifold and heat transfer cores have been proposed and tested during the last years. In the next sections a summary of the most promising geometries adopted for mHEX and mHS is presented with the aim to propose a common nomenclature for the description of these devices.

\section{THE HEAT TRANSFER CORE}

The heat transfer core of a mHEX or mHS can be structured in many different ways. As explained before, typically the core is obtained by stacking together a series of layers in which the flow is subdivided among parallel microchannels (MC core). The microchannels can be straight linear channels (Figure 4a) or nonlinear channels (Figure $4 \mathrm{~b}$ ) with or without a constant cross section. On the other hand the core can be obtained by using micropillars (PL core) displaced along the flow path in order to improve the heat transfer (Figure 4c).

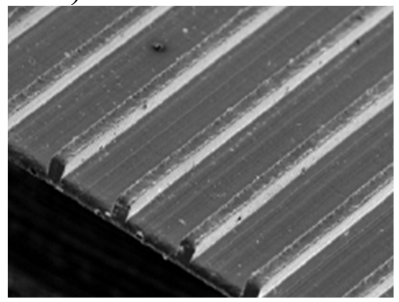

(a)

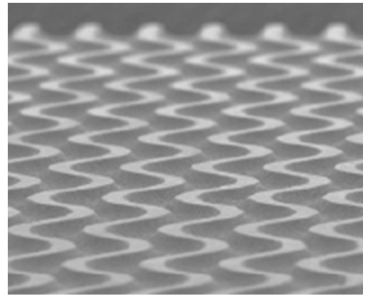

(b)

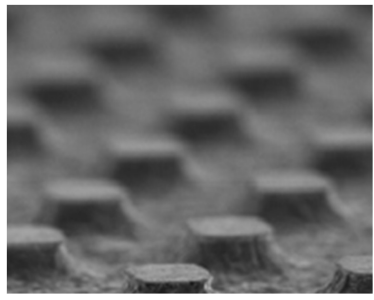

(c)

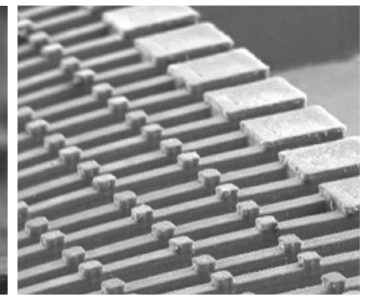

(d)

Figure 4. (a) Linear microchannels (MC core); (b) non-linear microchannels (MC core); (c) micro-pillars

(PL core); (d) Hierarchical channel network (HI core). 
The PL core is typically used if the fluid is introduced within the core orthogonally to the flow direction along the core (see Figure 4c). Hierarchical networks (HI core, see Figure 4d) can be also used with a local introduction of the fluid orthogonally to the heat transfer core.

The overall heat transfer coefficients obtained in the core depends strongly on the geometry adopted for the channels and on the flow arrangement.

Brandner et al. [7] have shown that PL cores tend to give higher overall heat transfer coefficients for fixed mass flow rate at the entrance with respect to MC cores, as evidenced by Figure 5. Staggered pillars are able to guarantee larger overall heat transfer coefficients with respect to in-line arrangements but, at the same time, an increase of the total pressure losses is expected. The results shown in Figure 5 highlight that the impact of the core geometry on the pressure losses and heat transfer coefficients can be very strong and an accurate design of a mHEX or mHS has always to start from an optimized design of the heat transfer core in which a trade-off between the values of pressure losses and of heat transfer coefficients is reached (see the green line of Figure 5).

Many geometries have been tested in the last decade, like non-uniform cross-section channels with periodic expansion-constriction cross-sections [20,21] and grooved channels [22]. The variation of the cross-section as well as the presence of secondary flows cause disruption in the hydrodynamic and thermal boundary layers by enhancing heat transfer and reducing pressure losses. However, the results reported in literature are in many cases contradictory.
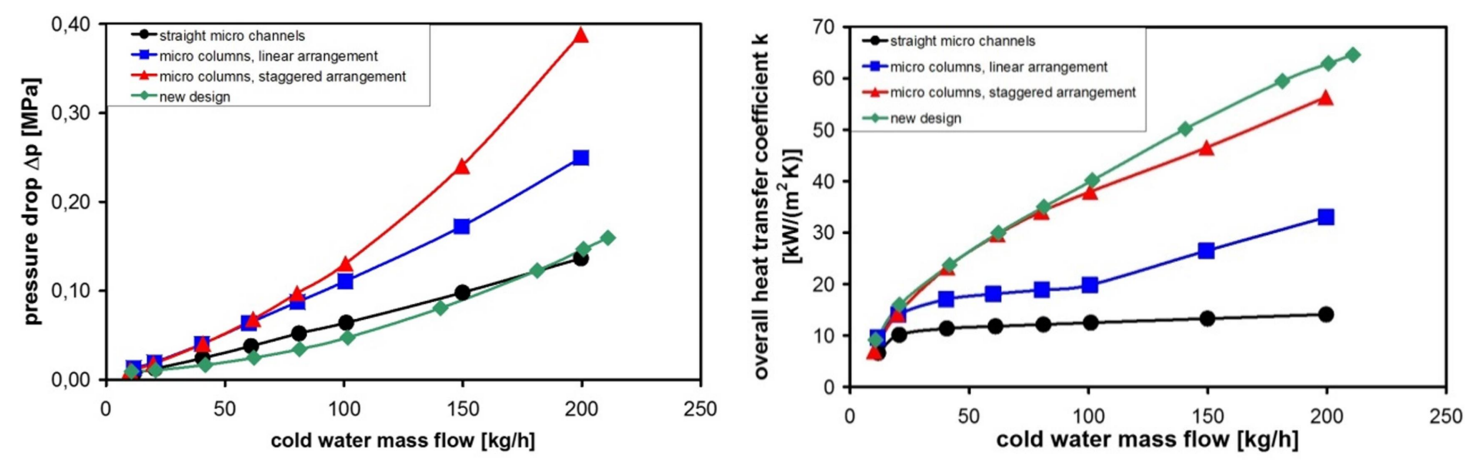

Figure 5. Pressure loss and overall heat transfer coefficient as a function of the water mass flow rate at the inlet of a mHEX for the four different configurations (see Fig 4a-d) of the mHEX core. From [7].

Many authors proposed the split-flow strategy in order to reduce pressure losses and to improve the heat transfer performances of mHS and mHEX [23-24]. In split-flow arrangement the flow can be introduced centrally and departed from two sides of the core, as shown in Figure 1c. This strategy can give some advantage with respect to the classical single-pass arrangement because the heat transfer core is divided in two or more regions and in this way: (i) the channel length is reduced (ii) the mass flow rate is reduced (iii) several developing regions are created with larger overall heat transfer coefficients. Microchannel networks (HI core) have been also explored in order to improve heat transfer [25,26].

Ghani et al. [27] in their extensive review about the effect of the channel geometry on the hydrothermal performances of microchannel heat sinks concluded that an optimal trade-off between heat transfer coefficients and pressure losses can be obtained by combining grooves, perforations and ribs in the heat transfer core of the device in order to enhance the presence of secondary flows.

To obtain a complete CFD numerical model of the heat transfer core of a mHEX obtained by means of a large numbers of microchannels and/or pillars can be very heavy in terms of CPU time due to the complex geometrical layout of the device. In order to solve this problem, many authors [28,29] have demonstrated that an accurate modelling of the heat transfer core can be obtained by assuming the core as a "regular" porous medium having a fixed porosity which can be calculated by knowing the geometry of the core. The results obtained with this approach, originally proposed by Kim and Kim [28], are satisfactory for many kind of mHEX and mHS. 


\section{MANIFOLDS OPTIMIZATION}

The presence of a large number of channels in parallel in the core of a mHEX or mHS generates the question of a correct distribution of the flow rate among the channels. This problem, usual also for conventional heat exchangers like plate heat exchangers of some importance, becomes crucial in micro heat exchangers and heat sinks because a true mal-distribution (MLD) of the mass flow rate among the parallel channels of a layer is responsible for a decrease of the overall performances of the device. For this reason, the problem of the optimisation of the geometry of the inlet and outlet regions before and after the heat exchanger core has been extensively investigated during the last decades. Two recent reviews on this topic are by Ghani et al. [30] and Siddiqui and Zubair [31]. Ghani et al. [30] divide the manifolds in: (i) dividing flow (Figure 6a) and (ii) combining flow (Figure 6b): usually the dividing manifold is the inlet manifold and the combining manifold is the outlet one. Other categories are indicated by Bajura and Jones [32] but these additional categories are related to the whole flow path within the device and will be recalled in the next paragraphs.
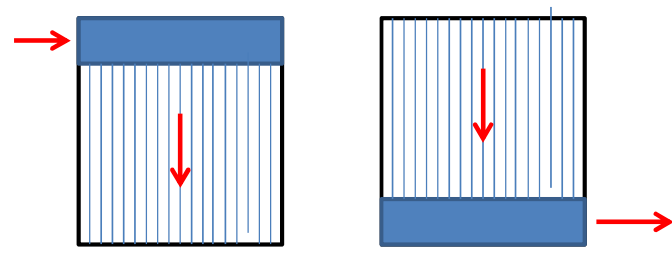

Figure 6. (a) Dividing manifold (inlet); (b) Combining manifold (outlet).

The analysis of the existing literature demonstrate that the position of the inlet and of the outlet ports as well as the shape of the manifolds at the inlet and at the outlet of a layer of parallel microchannels can strongly influence the flow rate distribution among the parallel channels.

For the manifolds, rectangular (R), trapezoidal (TP), triangular (T) shapes as well as the bifurcation type are the geometries most used and recommended for both mHEX and mHS. In Figure 7a a series of typical manifold shapes are shown. In Figure $7 \mathrm{~b}$ the most common locations of the inlet/outlet ports in correspondence of the manifolds are depicted; following the nomenclature reported in [30], three flow paths (C-type, V-type, I-type) can be obtained when the inlet and outlet ports are orthogonal to the mHEX core. On the contrary, if the inlet/outlet ports are in parallel to the core, three flow paths (Z-type, U-type, II-type) can be obtained. Of course, many other combinations of manifold shapes and inlet outlet port locations are possible.

By observing the flow path within the device, the arrangements shown in Figure 7 can be subdivided in three categories: (1) parallel flow arrangement in which inlet and outlet ports are located on the same side of the heat transfer core (C-type, U-type): in this case the length of the flow paths can be very different from one microchannel to another one; (2) reverse flow arrangement in which the inlet and the outlet ports are placed on different sides (V-type, Z-type): in this case the length of the possible flow paths between the inlet and the outlet are equalized; (3) normal flow arrangement in which the inlet/outlet ports are central with respect to the manifold length (I-type, II-type).

As indicated by Ghani et al. [27,30], it is not easy to navigate through the results obtained for mHEX and mHS because a shared taxonomy for the univocal description of a device was not defined. In order to overcome this problem, each device can be univocally identified by indicating:

(i) the core type (i.e. MC (microchannel core), HI (hierarchical core), PL (pillar core));

(ii) the manifold geometry (i.e. R (rectangular manifold), T (triangular manifold), TP (trapezoidal manifold), B (bifurcation manifold));

(iii) the flow path (i.e. I,II, C,V,Z,U-type flow path).

In this way, the specific layout of a mHS or mHEX can be univocally identified by means of a label $(\boldsymbol{E}, \boldsymbol{F}, \boldsymbol{G})$ in which $\boldsymbol{E}$ indicates the core type, $\boldsymbol{F}$ indicates the manifold geometry and $\boldsymbol{G}$ the flow path.

The large amount of publications reviewed in [30,31] underlines that the configuration and the shape of the manifolds influence the uniformity of flow distribution among the microchannels in particular when the 
number of microchannels is very large (i.e. $>20$ per layer). For this reason it becomes mandatory to optimize the configuration and the shape of the distributing/collecting manifolds of the device.

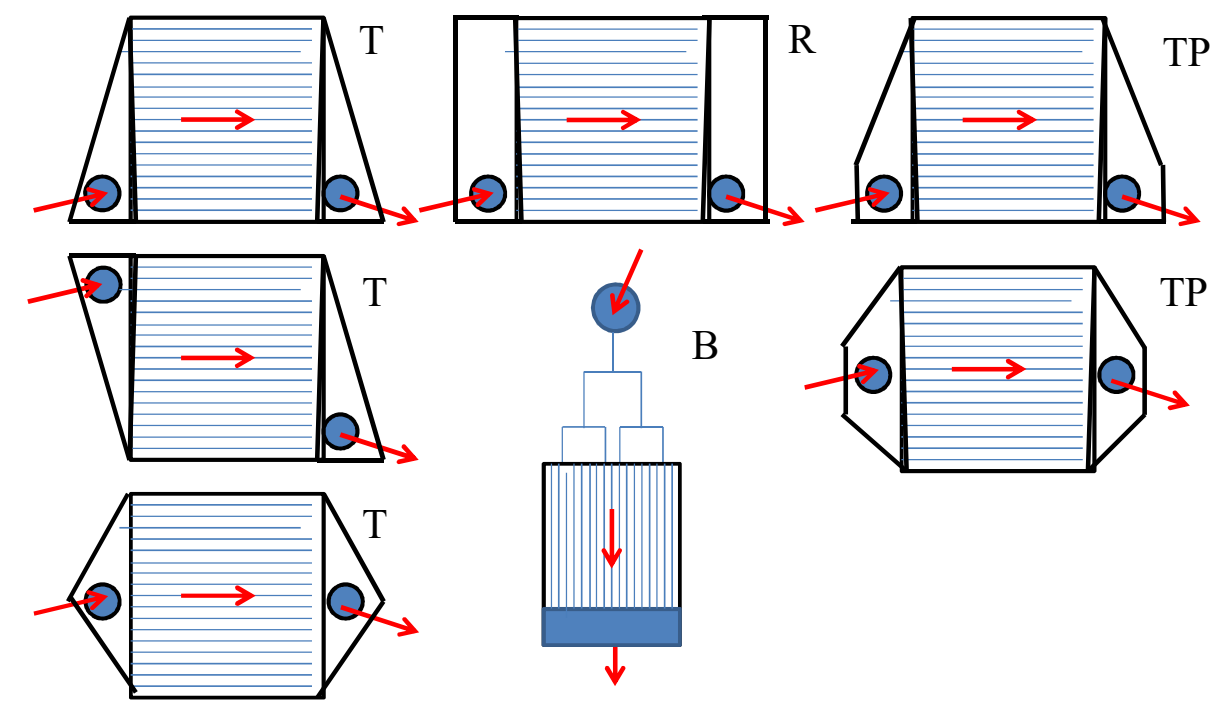

(a)

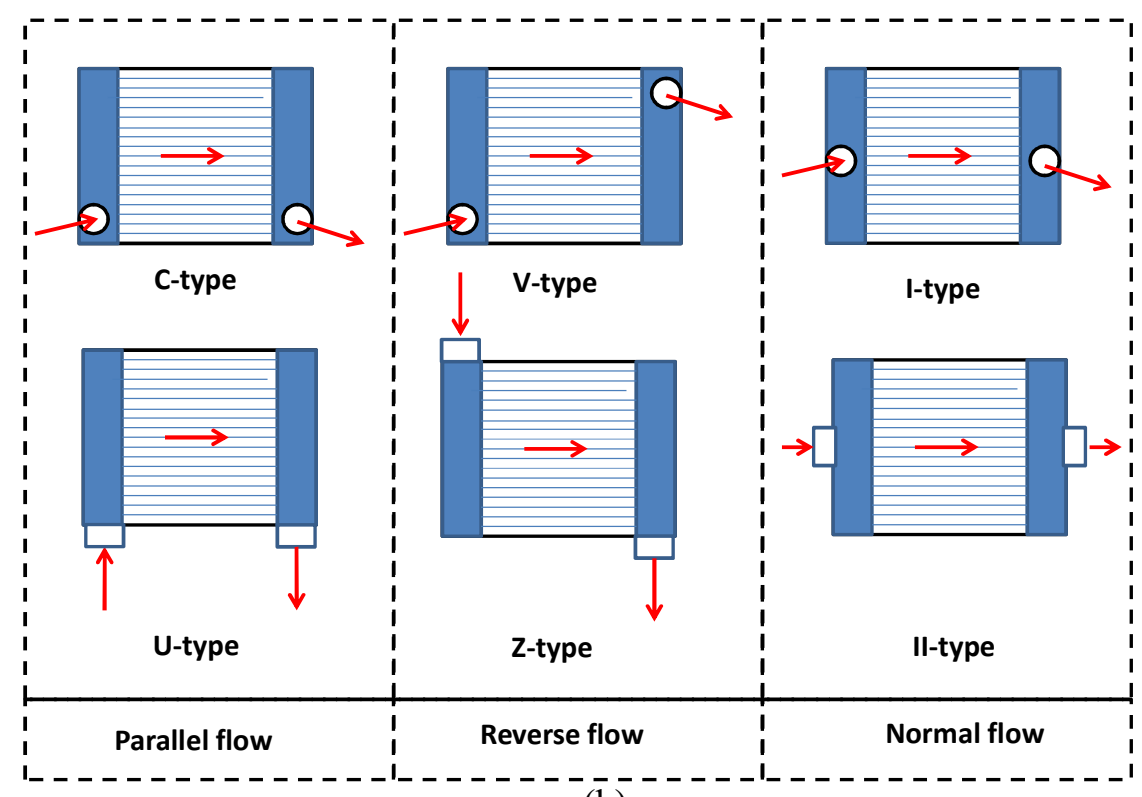

$(\bar{b})$

Figure 7. (a) Typical manifold geometries: rectangular (R), triangular (T), trapezoidal (TP), bifurcation (B); (b) Positions of the inlet/outlet ports: Orthogonal (C-type; V-type; I-type); In parallel (U-type; Z-type; IItype).

In order to demonstrate the effect of the geometry of the manifolds on the mass flow rate distribution, Yang et al. [13] studied numerically four different configurations of manifolds for a double-layer mHEX in order to check the possible influence of the manifold shape and configuration on the flow distribution among the channels of the single layer. In this case each layer contains $133(n)$ parallel linear microchannels (MC core) having a square cross section and a hydraulic diameter of $200 \mu \mathrm{m}$.

Four manifold configurations, shown in Table 2, have been tested. The inlet/outlet ports are orthogonal to the heat transfer core; following the nomenclature introduced previously, Case A, C and D generate a V-type flow path while Case B gives a typical C-type flow path.

By using the nomenclature proposed in this paper in ordr to describe the main features of each manifold configuration, Case A can be described as $(\mathrm{MC}, \mathrm{T}, \mathrm{V})$ which means that the mHEX has a microchannel core 
with two triangular manifolds with inlet/outlet ports orthogonal to the core (V-type) with a reverse flow path obtained by locating the inlet and outlet ports on the opposite sides of the dividing and combining manifolds. A group of small pillars with diameter of $0.8 \mathrm{~mm}$ have been added to the distribution/collecting manifold; the presence within the manifolds of supporting pillars or similar supporting structures is quite common in micro-devices (such as micro evaporators) where the dimensions and thickness of microstructures are greatly reduced in order to guarantee the mechanical stability of the device under high pressure.

Table 2. Geometry of the tested manifold configurations.

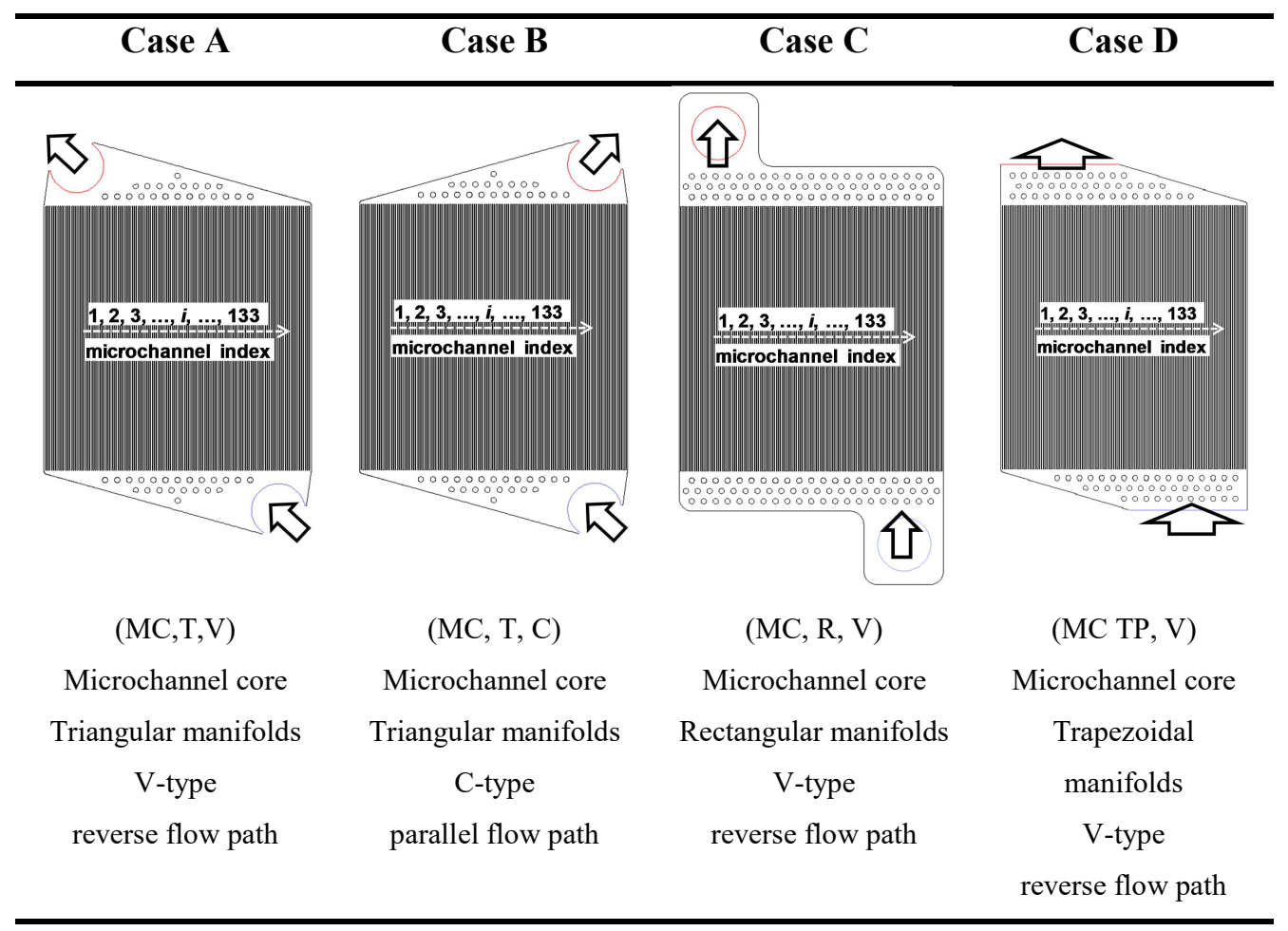

The position of the feeding ports in Case $\mathrm{C}$ and Case $\mathrm{D}$ is relatively far from the inlets of the microchannels, as shown in Table 2. The large area of the manifold and the large number of small pillars help to distribute the flow uniformly in the manifold before to enter into the core. In addition, Case D has enlarged feeding/exhaust ports in order to have smaller flow velocity at the same mass flow rate in the distributing/collecting chambers.

To know exactly how the different configurations of the manifold affect the flow distribution and to select the best one among the four configurations described in Table 2, all the 4 configurations have been numerically modelled by using the finite volume solver of ANSYS FLUENT ${ }^{\circledR}$.

The mass flow distribution through each microchannel has been determined as a function of the manifold configuration. The flow distribution was numerically examined for two different feeding pressures: $160 \mathrm{kPa}$ and $500 \mathrm{kPa}$. To have an insight into the mass flow distribution through each microchannel, the mass flow rate through every microchannel $\left(q_{i}\right)$ under different feeding pressures was calculated. The average mass flow rate $\mathrm{q}_{\mathrm{ave}}$ was calculated by means of the following equation:

$$
q_{\text {ave }}=\frac{1}{n} \sum_{i=1}^{n} q_{i}
$$

where $q_{i}$ is the mass flow rate of microchannel $i$.

Figure 8 shows the deviation of the mass flow rate through each microchannel from the average mass flow rate for Case A (a) and B (b). The microchannel index (i) increases from left to right. It is evident that in the Case A configuration (reverse flow), the mass flow rate is relatively uniform among the channels and close 
to the average value for microchannels in a broad middle section of the layer (Figure 8a). However, for microchannels close to the boundary of the heat exchanger core, the mass flow rate becomes larger or smaller than the average value. In addition, at larger mass flow rate or higher feeding pressure the flow distribution is less uniform through microchannels close to the lateral boundary of the core. However, in the middle part the flow distribution is much less influenced by the feeding pressure. This stable distribution is quite favorable for the performance of the microHEX.

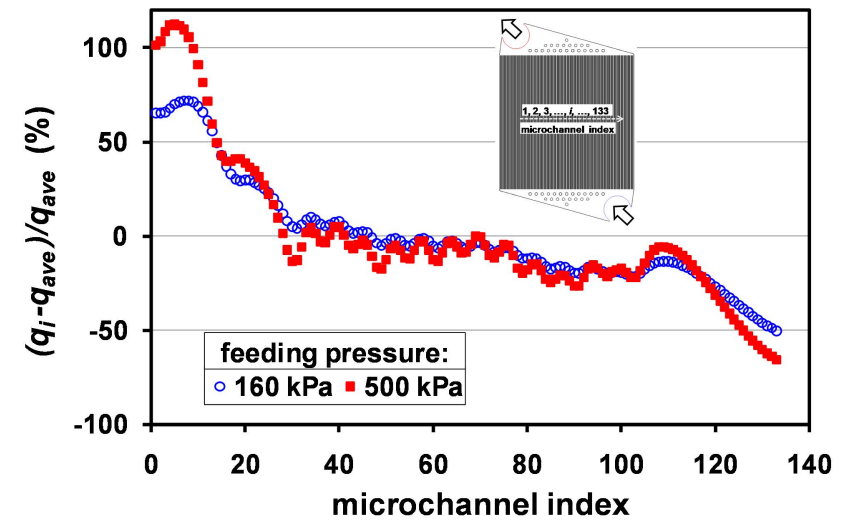

(a)

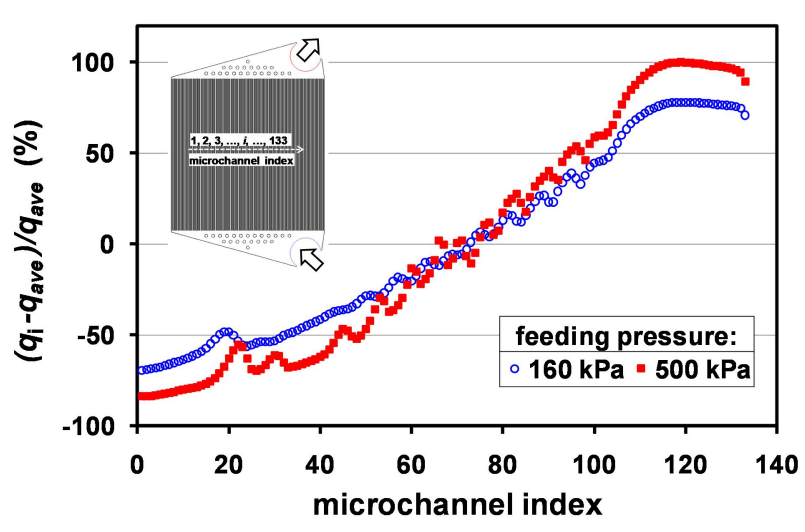

(b)

Figure 8. Mass flow distribution: (a) in a reverse flow path (Case A) and (b) in a parallel flow path (Case B).

As shown in Figure 8b, the parallel (C-type) flow path generated by Case B does not perform well in terms of uniform flow distribution among the parallel microchannels of the layer. From one side to the other of the layer, the mass flow rate through each microchannel increases almost linearly. Consequently, a uniform flow distribution can never be obtained by applying such kind of configuration. The simulation results suggest that a parallel flow path should always be avoided in the design of manifold configuration for microHEX if a nearly uniform distribution of mass flow rate is desired. Otherwise, with this kind of mass flow rate distribution one may obtain laminar flow in some microchannels and transitional flow in the others with unstable performances of the whole device.

Figure 9 shows the mass flow distribution among the parallel microchannels in Case C and D; in this case for both the geometries a reverse flow path is originated. It is evident that the rectangular shape of the manifolds (case C) is responsible of an asymmetric flow rate distribution having a parabolic shape, with lower flow rates in the middle section and larger flow rate near the boundaries of the layer.

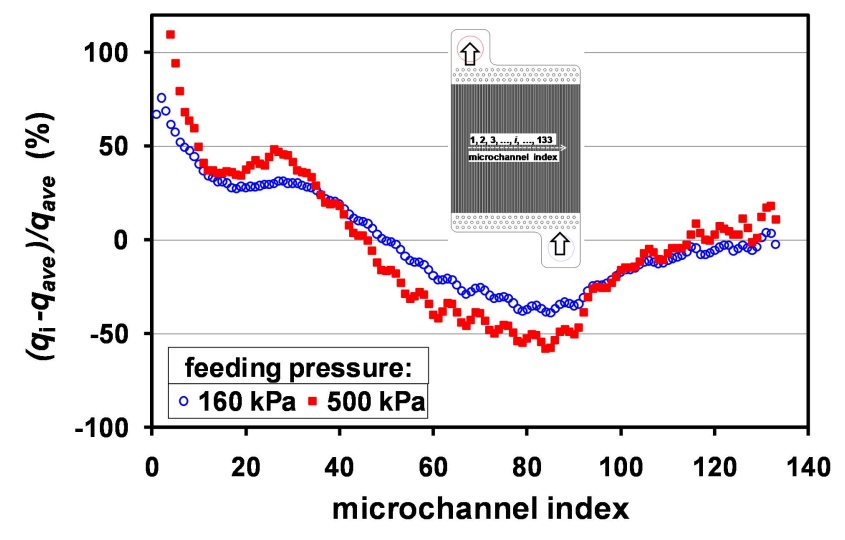

(a)

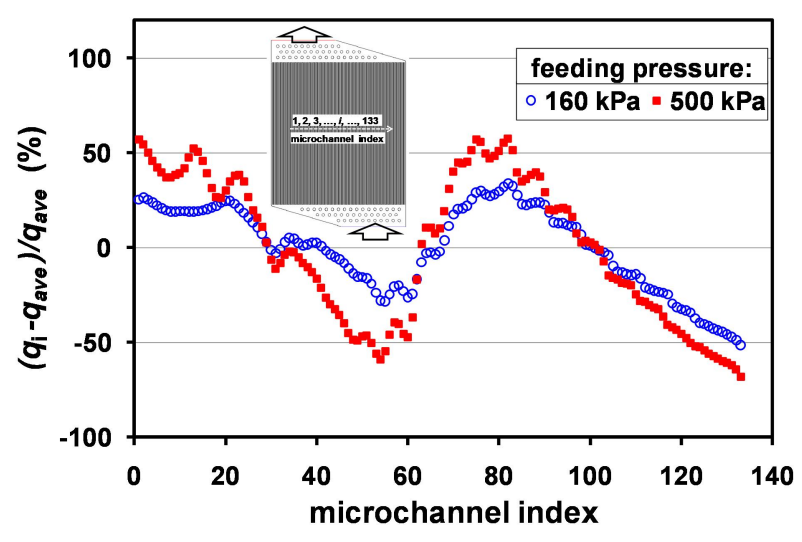

(b)

Figure 9. Mass flow distribution in the Case C (a) and D (b) configuration of the manifolds.

An enlargement of the inlet/outlet ports (Case D) generates non-uniform flow rate distribution throughout the whole layer, as highlighted by the data shown in Figure $9 \mathrm{~b}$. The mass flow rate decreases and increases 
sharply through neighbouring microchannels from one side to the other of the layer. By comparison between Case A and D, it seems that the enlargement of feeding/venting port leads to a negative effect on the flow distribution among the parallel channels.

In summary, a common conclusion for gas flows, confirmed by Figure 8 and 9, is that low values of the feeding pressure tends to improve the uniformity of the flow distribution among the parallel channels. By comparing the results of these four cases it is possible to conclude that Case A (MC,T,V) guarantees the best performances in terms of uniform distribution of the mass flow rate among the parallel microchannels, followed by Case C $(M C, R, V)$. The other two configurations (B: parallel flow path, D: enlarged inlet/outlet ports) are not able to generate uniformly distributed flow rates through the 133 parallel microchannels and they are not indicated for an optimized design of a microHEX.

The numerical results of Figure 8 and 9 have been obtained by means of a 2D model in which the real thickness of the manifolds, pillars and microchannels was not taken into account. The use of $2 \mathrm{D}$ models is very diffuse in the literature related to the optimization of the manifolds such as the work of Balaji and Lakshminarayanan [33], Commenge et al. [34], Tonomura et al. [35] and Renault et al. [36]. If the evaluation of the distribution of the mass flow rate among the channels is repeated by using a 3D model which takes into account the finite height of the layer the results are quite different. The flow distribution obtained from 2D and 3D numerical simulations is compared in Figure 10 for the same feeding pressure, which is fixed at $500 \mathrm{kPa}$ and for the Case A. It is evident that the flow distribution obtained by using the 3D model becomes more uniform than that obtained with the $2 \mathrm{D}$ model. This is due to the additional frictional drag on the bottom and top of the microchannels which is considered in the 3D model. Since by miniaturization the surface-to-volume ratio increases all the surface forces like the frictional ones becomes stronger and stronger and in this case are able to increase the uniformity of the flow rate distribution.

The mass flow distribution is plotted in Figure 10b for two feeding pressure values; it can be noted that the results obtained with the $3 \mathrm{D}$ model highlight that the flow distribution is less dependent on the feeding pressure. By observing the results of Figure $10 \mathrm{~b}$, the relative deviation of flow rate is almost the same through more than 100 microchannels for the two considered values of the feeding pressure.

It is possible to conclude that a $2 \mathrm{D}$ model of the manifolds tends generally to overestimate the flow maldistributions in micro heat sinks and micro heat exchangers.
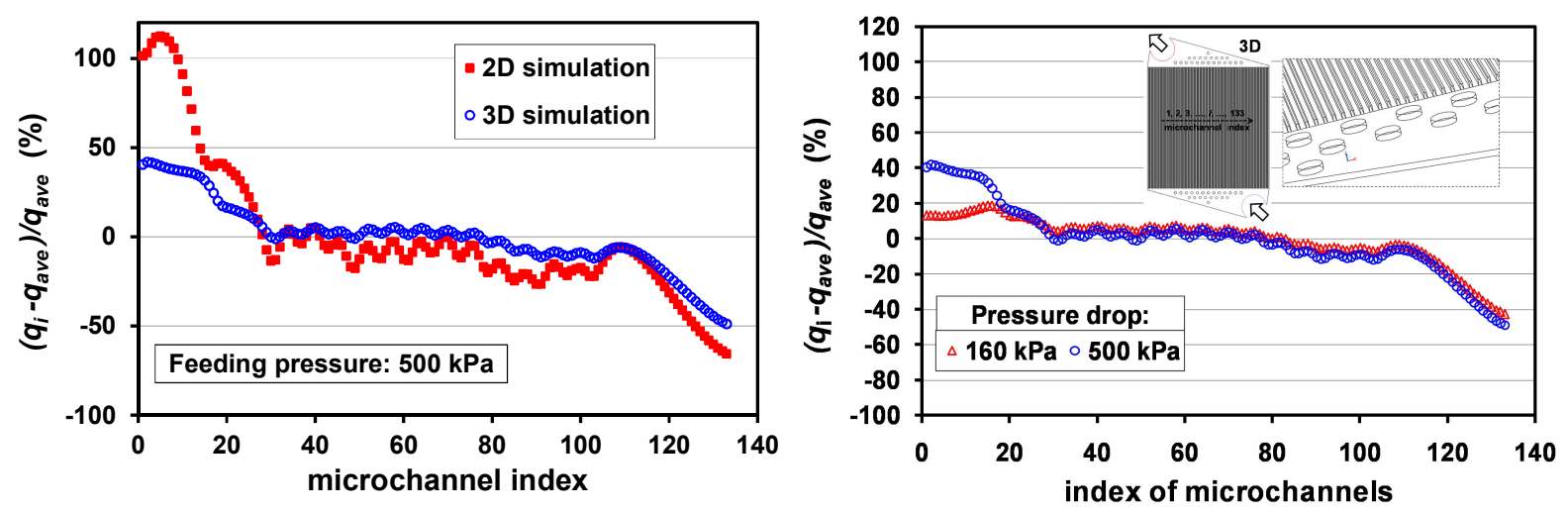

Figure 10. Comparison of flow distribution obtained from 2D and 3D models for manifolds Case A.

These results are confirmed by a series of other experimental and numerical observations summarized by Ghani et al. [30]. As a rule of thumb, it is possible to conclude that, from a fluid-dynamic point of view, reverse flow path arrangement is generally preferable to the other flow paths due to the uniformity of the flow paths guaranteed by the position of the inlet/outlet ports. As observed by Ghani et al. [30] reverse flow arrangements provide better flow distribution and lower pressure losses than parallel flow arrangement. Other general conclusions, partially derived by the review of Ghani et al. [30], are the following:

(i) for orthogonal inlet/outlet ports, the I-type normal flow path arrangement seems to provide better flow distribution than other types of flow arrangements in presence of rectangular manifolds for a limited number of parallel channels per layer $(n<30)$; 
(ii) in presence of a large number of parallel channels $(n>30)$ the V-type configuration with a reverse flow path must be selected in order to provide an uniform distribution of the flow rate in combination with triangular manifolds;

(iii) an increase in the inlet port area reduces the effect of jet flow at the entrance and enhance the uniformity of the flow distribution among the parallel channel; however, an enlargement of feeding/venting ports leads to a negative effect on the flow distribution in presence of a large number of parallel channels $(n>30)$.

(iv) the total cross sectional area of the heat transfer core in presence of microchannels must be lower than manifold area for superior performances;

(v) an increase of the manifold length at fixed width provides better thermal performances;

(vi) the area of combining manifold must be larger than the area of dividing manifold.

For sake of completeness, other techniques proposed in literature in order to obtain more uniform distribution of the flow rate among the channels of the core region of a mHS or mHEX are the following:

1) the introduction of a bifurcation-type distribution system (B in Figure 7a) at the inlet of the parallel channels [37] can guarantee a more uniform distribution of the flow rate by driving the repartition of the flow rate thanks to a treelike network (see Figure 11a) designed by following the Bejan's law of the constructal theory. Brandner and Maikowske [37] demonstrated that this arrangement is able to eliminate mal-distributions even in presence of two phase flows (i.e. evaporators). Negative aspects of this solution are: (i) the increase of the device volume; (ii) complex 3D manufacturing; (iii) strong increase of the device total pressure losses determined by the treelike network and thus it becomes unsuitable for reduced pressure drop related applications.

2) the introduction of a metallic foam within the inlet and outlet manifolds by means of which a more uniform distribution of the working fluid can be obtained thanks to the random distribution of the pores of the metallic foam (Gong et al. [38]). Also in this case the presence of the metallic foam increases the total pressure losses of the device.

3) the introduction of micro-orifices at the inlet of a set of parallel microchannels (see Figure 11b) can enable to equalize the flow rate distribution in the core of a mHS or mHEX as demonstrated by Szczukiewicz et al. [39]. The authors demonstrated experimentally that by using inlet restrictions in each microchannel of a micro-evaporator, flow instabilities, vapor back flow, and flow maldistribution can be avoided.

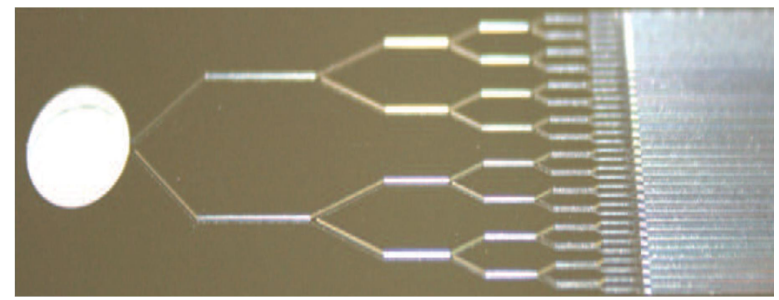

(a)

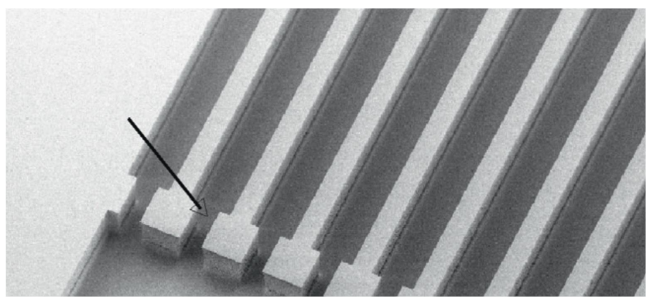

(b)

Figure 11. (a) Bifurcation distribution system proposed by Brandner and Maikowske [37]; (b) inlet restrictions proposed by Szczukiewicz et al. [39]

In conclusion, it is important to highlight that the optimization of the manifolds is not only important in order to mitigate the mal-distribution of the flow rate among the parallel microchannels which are present in the core of the mHS or mHEX but also to reduce instabilities and the presence of flow dead zones within the device. The presence of regions in which the flow velocity is very low (stagnation regions) can be very dangerous for a rapid growth of the fouling. In fact, as observed by Mayer et al. [40] the impact of crystallization fouling depends strongly on flow velocity and local surface temperature; for this reason, the presence of flow dead zones, particularly in the distributors, need to be avoided in mHEX and mHS.

\section{EFFECTS OF CONJUGATE WALL-FLUID HEAT TRANSFER}

In mHS and mHEX the thickness of the solid walls can be comparable with the inner dimensions of the micro channels. As a consequence, the thermal interaction between fluid and solid walls cannot be ignored 
when heat transfer is activated. Many publications showed that conjugate heat transfer is able to affect the performances of the heat exchangers. As demonstrated by means of a series of experimental results $[13,14,41,42]$ the effect on the heat exchanger effectiveness of the conjugate heat transfer changes with the adopted flow configuration (i.e. counter-flow, co-current flow, cross flow).

As an example, the effect of the conjugate wall-fluid heat transfer in presence of thick solid walls on the effectiveness of a conventional counter-current heat exchanger has been extensively investigated and the following expression of the effectiveness can be found in literature [42]:

$$
\varepsilon=1-\frac{1}{1+N T U\left(\frac{1+\lambda \Phi}{1+\lambda N T U}\right)}
$$

in which the longitudinal conduction parameter $\lambda[41,42]$ appears, defined as the ratio of the axial conductive heat flux in wall $\left(\phi_{\mathrm{w}}\right)$ and the convective heat flux of the fluid $\left(\phi_{\mathrm{f}}\right)$ :

$$
\lambda=\frac{\phi_{w}}{\phi_{f}}=\frac{k_{w} A_{w} / L}{q_{t o t} c_{p}}
$$

where $k_{w}$ is the thermal conductivity of the solid wall, $A_{w}$ the cross sectional area of the wall, $L$ is the channel length, $q$ the mass flow rate and $c_{p}$ the specific heat of the fluid. The parameter $\Phi$ is defined as:

$$
\Phi=\sqrt{\frac{\lambda N T U}{1+\lambda N T U}}
$$

Eq.(8) highlights that the conjugate heat transfer between the solid wall and the fluid tends to reduce the value of the heat exchanger effectiveness. This reduction is stronger for larger NTU values, which means in presence of larger overall heat transfer coefficients and lower mass flow rates: this is exactly the case of mHEX and mHS. Eq.(8) suggests to use the conduction parameter $\lambda$ as parameter for checking the role of the conjugate heat transfer in a mHEX or mHS. When the conduction parameter tends to zero, Eq.(8) gives the well-known expression of the effectiveness for a counter-current heat exchanger with balanced flow $(\varepsilon(\lambda=0)=1-1 /(1+N T U))$. As rule of thumb, in coherence with the suggestion of Morini and Yang [16], when $\lambda<0.01$ the conjugate effects can be ignored in the design of a mHEX or mHS and the conventional $\varepsilon$-NTU curves developed by ignoring this effect can be used.

Let's consider a multi-layered heat exchanger in which the heat transfer core is composed by 10 layers of 133 squared microchannels $\left(D_{h}=200 \mu \mathrm{m}\right)$ of fixed cross section (MC core). The adjacent layers are separated by a partition foil having a fixed value of thermal conductivity $\left(k_{w}\right)$ and a fixed thickness of $100 \mu \mathrm{m}$.

A 3D numerical simulation has been carried out for the multi-layered mHEX in order to check the effect of the thermal conductivity of the partition foil on the effectiveness of the mHEX assuming counter-current flow arrangement and adopting balanced mass flow rates at the inlets. The mHEX is calculated as a function of the partition foil thermal conductivity, as shown in Figure 12. It is evident that the effectiveness of the multi-layered mHEX depends on the thermal conductivity of the partition foil and the trend shows that usually an optimal value of $k_{w}$ exists in order to maximize the heat exchanger effectiveness. The trend of Figure 12 is similar to that obtained numerically by Stief et al. [43]; in this specific case, a flatter region close to the maximum value of effectiveness is observed which means that an interval of thermal conductivity values can be considered being able to maximize the mHEX performances. The range of partition foil thermal conductivity linked to optimal effectiveness can be narrowed by increasing the partition foil thickness.

The result shown in Figure 12 is very general; however, similar trends can be observed for all the flow configurations, except for the case of the co-current flow arrangement, as demonstrated below.

The presence of a maximum value of effectiveness is due to the trade-off between the heat transferred across the partition wall and the heat transferred by axial conduction along the partition wall. In fact, if a low value of thermal conductivity of the partition wall is adopted the heat transfer between the fluids is reduced and hence the effectiveness tends to zero for $k_{w}$ tending to 0 ; on the contrary, for large values of $k_{w}$ heat will be transferred mainly for axial conduction along the partition wall from the hot to the cold region of the mHEX by reducing the effectiveness of the heat exchanger. This means that it becomes possible to optimize the mHEX effectiveness by selecting a specific material as partition foil. 
Of course, the heat exchanger effectiveness is not merely influenced by the thermal conductivity of the partition foil but it is also correlated to the thermal properties of the working fluids. In other words, the same mHEX may have completely different performance if the working fluid is changed from gas to liquid. Figure 12 puts in evidence in which way the trend of the effectiveness is influenced by the properties of the working fluids. It is interesting to observe that the range of conductivity corresponding to the maximum effectiveness is greatly shifted if the working fluid is changed from air to water. For instance, if glass (thermal conductivity around $1 \mathrm{~W} /(\mathrm{mK}))$ is used to manufacture the $\mathrm{mHEX}$, the device will have an effectiveness of $78 \%$ with air flows but only $50 \%$ if water is used as the working fluid.
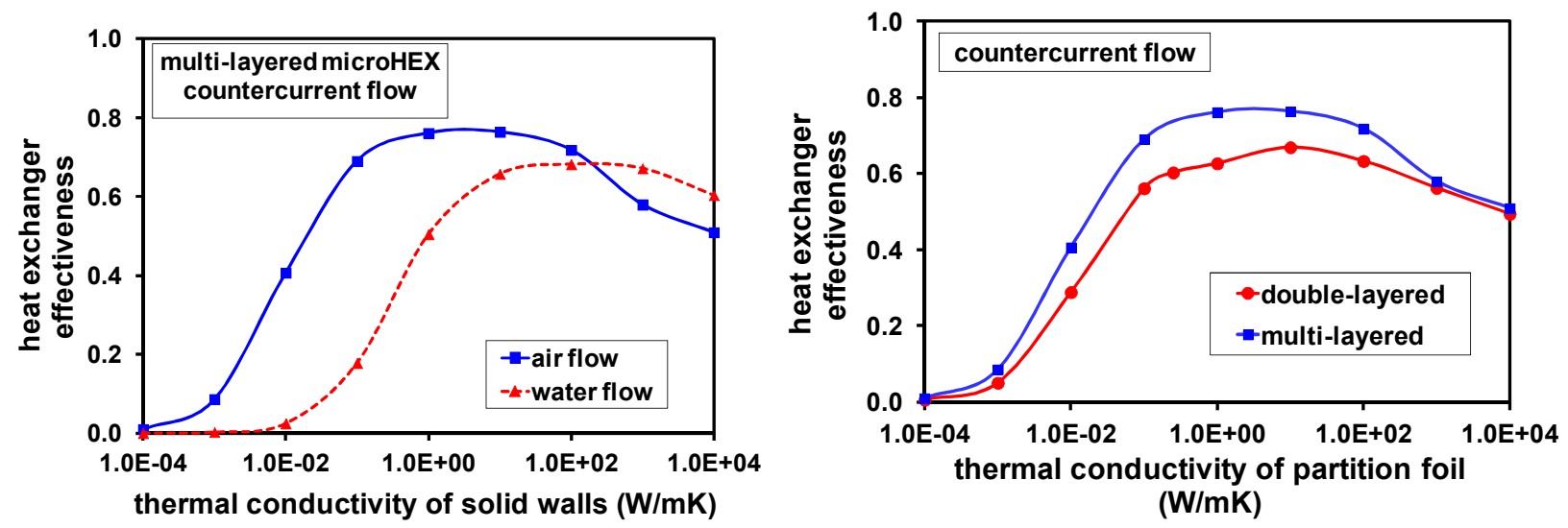

Figure 12: Heat exchanger effectiveness as a function of wall thermal conductivity for a multi-layered microHEX under counter-current flow configuration: (a) effect of the working fluid; (b) effect of the number of layers.

The effect of the number of layers on the mHEX effectiveness is shown in Figure 12b; usually, by increasing the number of layers the effectiveness increases for a fixed value of the Reynolds number (in this case $\mathrm{Re}=550$ with air as working fluid). It is evident that the difference in terms of effectiveness is very small for partition foils with very low or very high thermal conductivity. However, in the region in which the effectiveness is close to its maximum value, which is the region where the partition foil thermal conductivity is between $0.1 \mathrm{~W} /(\mathrm{mK})$ and $100 \mathrm{~W} /(\mathrm{mK})$, the difference in terms of effectiveness becomes fairly large. In this range, the effectiveness of the double-layered device is around $10 \%$ lower than that of the multi-layered one.

Finally, in Figure 13 the thermal performances of a double-layered mHEX are compared with those of a multi-layered mHEX by considering a co-current flow arrangement. Similar to the case of a countercurrent flow arrangement, the multi-layered mHEX is characterized by values of effectiveness larger than the values obtained for the double-layered mHEX. For values of the wall thermal conductivity very low (lower than 0.1 $\mathrm{W} /(\mathrm{mK}))$, the difference in terms of effectiveness between the two devices is low as in the case of the counter-current flow arrangement. On the contrary, for large values of the wall thermal conductivity (i.e. larger than $1 \mathrm{~W} /(\mathrm{mK}))$, the conductivity of the partition foil is not able to influence the effectiveness of the mHEX. No maximum is observed for co-current configuration. In the case of the multi-layered mHEX, when the foil thermal conductivity becomes larger than $1 \mathrm{~W} /(\mathrm{mK})$ the effectiveness tends to its asymptotic value of $50 \%$. This result is in agreement with the expected asymptotic value of the effectiveness of a co-current heat exchanger when NTU tends to $\infty$.

The trend of the mHEX effectiveness of Figure 13 obtained for a co-current flow arrangement can be explained by observing that, in the case of balanced co-current flows of the same fluid on the cold and hot side of the heat exchanger, the cold flow local temperature rise is ideally equal to the hot flow local temperature decrease. For this reason, the partition foil between the two fluids works in this case at a value of temperature equal to the average value between the hot and the cold flow. As a result, the temperature of the partition foil tends to be axially uniform along the microHEX and for this reason no wall axial conduction can exist even in the case of partition foils with a large thermal conductivity. Therefore, it can be concluded that wall axial conduction loses importance in the case of co-current flow arrangement. 
This observation explains the experimental results obtained by Koyama and Asako [11] obtained for gas-togas micro heat exchangers.

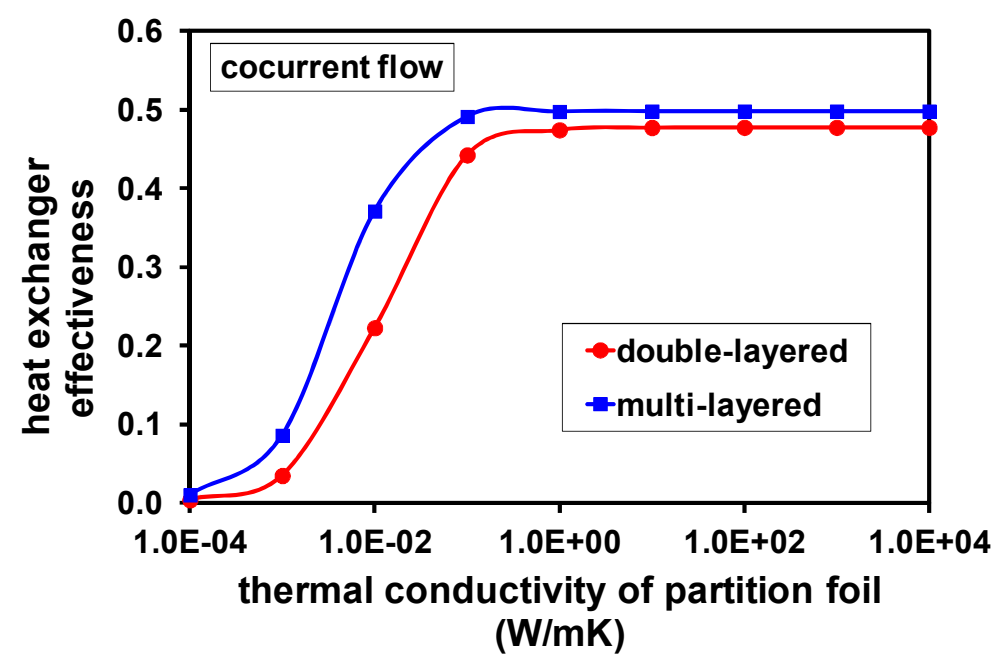

Figure 13. Comparison on thermal performance between double-layered and multi-layered microHEXs for co-current flow arrangement.

The results shown in this section suggest that, especially in presence of gaseous working fluids, when the heat transfer core is obtained by using mini or micro-channels high effectiveness can be obtained even in presence of solid walls characterized by low values of thermal conductivity. This observation opens to the application of "chip" material (i.e. plastic materials) for the fabrication of mHEX and mHS with the possibility to extend the range of microfabrication techniques used for the production of these devices, like $\mathrm{CNC}$ desktop milling machines and 3D printers. This result can allow a more rapid diffusion of these devices also for specific applications which need customized solutions

One of the fields in which this result has been already tested and applied is air conditioning. Nowadays, it becomes possible to build counter-current heat recovery devices for exhaust air in mechanical ventilation systems by using mini-channels in plastic materials by saving money and weight without to have a heavy penalty in terms of efficiency.

\section{CONCLUSIONS}

Micro heat exchangers and heat sinks are promising devices able to transfer large amount of heat in a reduced volume. This characteristic enables their use in many technological fields in which the compactness is an added value like in the automotive industry, aerospace, electronic cooling or biomedical applications. Even if many imaginative configurations have been proposed and tested for both micro heat exchangers and heat sinks during the last two decade, a complete overview of the results accumulated up to now is only partially available and an effort in order to coordinate the studies in this field was not made until now. For example, a common taxonomy to describe the different arrangements and flow configurations of these devices was never proposed yet. Even if in literature some review papers on this topic are available, the efforts to obtain a common frame in which all the published results can be organized are still insufficient. Patents slowed down the starting of a systematic rereading of the existing results. In addition, many published results seem to be incoherent with each other.

However, by analyzing the results collected until now a series of general conclusions and design rules can be made.

- the design of micro heat exchangers can be obtained by using the conventional methods developed for classical heat exchangers even if the presence of non- negligible scaling effects (i.e. compressibility effects, conjugate wall-fluid effects, viscous dissipation) must be always verified; when these scaling effects are present they can become an opportunity in order to differentiate the features of mHEX and mHS from the conventional devices. However, in presence of significant scaling effects the use of conventional charts (i.e. $\varepsilon$-NTU curves) cannot guarantee the proper design of these components. In this paper it is highlighted that the presence of strong conjugate wall-fluid heat transfer effects in mHEX can offer the opportunity to use materials having low thermal conductivity values (i.e. plastic materials), 
especially in presence of counter-current flow and cross-flow configurations. This fact enables the use of innovative fabrication techniques (i.e. 3D printers) which can reduce the costs of a customized fabrication of mHEX and $\mathrm{mHS}$.

- the performances of mHEX and mHS are strongly influenced by the proper distribution of the flow rate within the heat transfer core and a series of different solutions are available in order to solve this problem which are summarized in this paper.

Further efforts, especially a homogenization of taxonomy and experimental description, are needed in order to obtain a complete overview of the main design rules of these interesting devices.

\section{ACKNOWLEDGMENTS}

This work has been granted by MIGRATE, a Marie-Skłodowska-Curie Innovative Training Network (ITN). This ITN Research Project is supported by the European Union Horizon 2020 Programme Funding under the Grant Agreement No. 643095 (call H2020-MSCA-ITN-2014).

\section{NOMENCLATURE}

$\begin{array}{llllll}d & \text { channel diameter } & (\mathrm{m}) & N T U & \text { number of transfer units } & (-) \\ D_{h} & \text { hydraulic diameter } & (\mathrm{m}) & q & \text { mass flow rate } & (\mathrm{kg} / \mathrm{s}) \\ F & \text { correction factor } & (-) & \varepsilon & \text { heat exchanger effectiveness } & (-) \\ k & \text { overall heat transfer coefficient } & \left(\mathrm{W} / \mathrm{m}^{2} \mathrm{~K}\right) & \Phi & \text { parameter defined by Eq. }(10) & (-) \\ k_{w} & \text { wall thermal conductivity } & (\mathrm{W} / \mathrm{mK}) & \lambda & \text { parameter defined by Eq. }(9) & (-) \\ L & \text { channel length } & (\mathrm{m}) & & & \\ n & \text { number of channels } & (-) & & & \end{array}$

\section{REFERENCES}

[1] Morini, G.L., "Single-phase Convective Heat Transfer in Microchannels: a Review of Experimental Results", Int. J. of Thermal Sciences, 43, pp. 631-651, (2004).

[2] Hessel, V., Schouten, J.C., Renken, A., Yoshida, J.I. eds, Handbook of Micro Reactors: Chemistry and Engineering, Weinheim: Wiley-VCH Verlag GmbH \& Co., (2009)

[3] Schubert, K., Brandner, J.J., Fichtner, M., Linder, G., Schygulla, A., Wenka, A., "Microstructure devices for applicationsin thermal and chemical process engineering", Microscale Thermophysical Engineering, 5(1), pp. 17-39, (2001).

[4] Dixit, T., Ghosh, I., "Review of micro- and mini-channel heat sinks and heat exchangers for single phase fluids", Renewable and Sustainable Energy Reviews, 41, pp. 1298-1311, (2015).

[5] Ahmed, H.E., Salman, B.H., Kherbeet, A.S., Ahmed, M.I., "Optimization of thermal design of heat sinks: a review", International Journal of Heat and Mass Transfer, 118, pp. 129-153, (2018).

[6] Harris, C., Kelly, K., Wang, T., McCandless, A., Motakef, S., "Fabrication modeling and testing of micro-cross-flow heat exchangers", Journal of Microelectromechanical Systems, 11(6), pp. 726-735, (2002).

[7] Brandner, J.J., Anurjew, E., Bohn, L., Hansjosten, E., Henning, T., Schygulla, U.,Wenka, A., Schubert, K., "Concepts and realization of microstructure heat exchangers for enhanced heat transfer", Experimental Thermal Fluid Science, 30(8), pp.801809, (2006).

[8] Alm, B., Imke, U., Knitter, R., Schygulla, U., Zimmermann, S., "Testing and simulation of ceramic micro heat exchangers", Chemical Engineering Journal, 135(1) pp. S179-S184, (2008).

[9] Garcia-Hernando, N., Acosta-Iborra, A., Ruiz-Rivas, U., Izquierdo, M., "Experimental investigation of fluid flow and heat transfer in a single-phase liquid flow micro-heat exchanger", International Journal Heat Mass Transfer, 52 pp.5433-5446, (2009)

[10] Bier, W., Keller, W., Linder, G., Seidel, D., Schubert, K., Martin, H., "Gas to gas heat transfer in micro heat exchangers", Chemical Engineering Process: Process Intensification, 32(1), pp.33-43, (1993).

[11] Meschke, F., Riebler, G., Hessel, V., Schuerer, J., Baier, T., "Hermetic gas-tight ceramic microreactors", Chemical Engineering Technology, 28(4), pp. 465-473, (2005).

[12] Koyama, K., Asako, Y., "Experimental investigation of heat transfer characteristics on a gas-to-gas parallel flow microchannel heat exchanger", Experimental Heat Transfer, 23, pp. 130-143, (2010).

[13] Yang, Y., Gerken, I. Brandner, J.J., Morini, G.L., "Design and Experimental Investigation of a Gas Flow Micro Heat Exchanger", Experimental Heat Transfer, 27 (4), pp. 340-359 (2014) 
[14] Yang, Y., Morini, G.L., Brandner, J.J., "Experimental analysis of the influence of wall axial conduction on gas-to-gas micro heat exchanger effectiveness", International Journal Heat and Mass Transfer, 69, pp. 17-25 (2014)

[15] Zimmermann, S., Meijer, I., Tiwari, M.K., Paredes, S., Michel, B., Poulikakos, D., "Aquasar: A hot water cooled data center with direct energy reuse", Energy 43(1), pp. 237-245, (2012).

[16] Morini, G.L., Yang, Y., "Guidelines for the analysis of single-phase forced convection in microchannels", ASME Journal of Heat Transfer, 135, 101004 (2013).

[17] Chen, X., Su, Y., Reay, D., Riffat, S., "Recent research developments in polymer heat exchangers - a review", Renewable and Sustainable Energy Reviews, 60, pp. 1367-1386 (2016).

[18] Gnielinski, V., "Ein Neues Berechnungsverfahren fur die Warmeubertragung im Ubergangsbereich zwischen Laminaren und Turbulenter Rohstromung [A New Calculation Procedure for the Heat Transfer in the Transition Region Between Laminar and Turbulent Pipe Flow]", Forschungim Ingenieurwesen-Engineering Research, 61, pp. 240-248, (1995).

[19] Palm, B., "Heat transfer in microchannels", Microscale Thermophysical Engineering, 5(3), pp. 155-175, (2001)

[20] Chai, L., Xia, G., Wang, L., Zhou, M., Cui, Z., "Heat transfer enhancement in microchannel heat sinks with periodic expansion-constriction cross-sections", International Journal Heat Mass Transfer, 62, pp. 741-751, (2013).

[21] Chai, L., Xia, G., Zhou, M., Li, J., "Numerical simulation of fluid flow and heat transfer in a microchannel heat sink with offset fan-shaped reentrant cavities in sidewall”, International Communication Heat Mass Transfer, 38(5), pp. 577-584, (2011).

[22] Herman, C., Kang, E., "Heat transfer enhancement in a grooved channel with curved vanes", International Journal Heat Mass Transfer, 45, pp. 3741-3757, (2002).

[23] Vinodhan, V.L., Rajan, K., "Computational analysis of new microchannel heat sink configurations", Energy Conversion and Management, 86, pp. 595-604, (2014).

[24] Tan, S., Toh, K.C., Kahn, N., Pinjala, V., Kripesh, V., "Development of single-phase liquid cooling solution for 3-D silicon modules", IEEE Transaction Components Packaging Manufacturing Technology, 1, pp. 536-544, (2011).

[25] Xie, G., Li, S., Sunden, B., Zhang, W., Li, H., "A numerical study of the thermal performance of microchannel heat sinks with multiple length bifurcation in laminar liquid flow", Numerical Heat Transfer Part A, 65(2), pp. 107-126, (2014).

[26] Muzychka, Y.S., "Constructal design of forced convection cooled microchannel heat sinks and heat exchangers", International Journal Heat Mass Transfer, 48, pp. 3119-3127, (2005).

[27] Ghani, I.A., Sidik, N.A.C., Kamaruzaman, N., "Hydrothermal performance of microchannel heat sink: the effect of channel design”, International Journal Heat Mass Transfer, 107, pp. 21-44, (2017).

[28] Kim, S.J., Kim D., "Forced convection in microstructures for electronic equipment cooling", ASME Journal of Heat Transfer, 121, pp. 639-645 (1999).

[29] Hatami, M., Ganji, D.D., "Thermal and flow analysis of microchannel heat sink (MCHS) cooled by Cu-water nanofluid using porous media approach and least square method", Energy Conversion and Management, 78, pp. 347-358, (2014).

[30] Ghani, I.A., Sidik, N.A.C., Kamaruzaman, N., Yahya, W.J., Mahian, O., "The effect of manifold zone parameters on hydrothermal performance of micro-channel heat sink: a review", International Journal Heat Mass Transfer, 109, pp. 11431161, (2017).

[31] Siddiqui O.K., Zubair, S.M., "Efficient energy utilization through proper design of microchannel heat exchanger manifolds: a comprehensive review", Renewable and Sustainable Energy Reviews, 74, pp. 969-1002 (2017).

[32] Bajura, R., Jones, E., "Flow distribution manifolds", ASME Journal Fluids Engineering, 98, pp. 654-665 (1976).

[33] Balaji, S., Lakshminarayanan, S., "Improved design of microchannel plate geometry for uniform flow distribution," The Canadian Journal of Chemical Engineering, 84(6), pp. 715-721, (2006).

[34] Commenge, J.M., Falk, L., Corriou, J.P., Matlosz, M., "Optimal design for flow uniformity in microchannel reactors," AIChE Journal, 48(2), pp. 345-358, (2002).

[35] Tonomura, O., Tanaka, S., Noda, M., Kano, M., Hasebe, S., Hashimoto, I., "CFD-based optimal design of manifold in plate-fin microdevices," Chemical Engineering Journal, 101, pp. 397 - 402, (2004).

[36] Renault, C., Colin, S., Orieux, S., Cognet, P., Tzedakis, T., "Optimal design of multi-channel microreactor for uniform residence time distribution", Microsystem Technologies, 18, pp. 209-223, (2012).

[37] Brandner, J.J., Maikowske, S., "Influence of Fluid Flow Distribution in Micro-Channel Arrays to Phase Transition Processes", Experimental Heat Transfer, 25(3), pp. 172-180, (2012)

[38] Gong., L., Zhao, J., Huang, S., "Numerical study on layout of micro-channel heat sink for thermal management of electronic devices", Applied Thermal Engineering, 88(5), pp. 480-490, (2015)

[39] Szczukiewicz, S., Borhani, N., Thome, J.R., "Two-phase flow operational maps for multi-microchannel evaporators", International Journal of Heat and Fluid Flow, 42, pp. 176-189, (2013)

[40] Mayer, M., Bucko, J., Benzinger, W., Dittmeyer, R., Augustin, W., Scholl, S., "The impact of crystallization fouling on a microscale heat exchanger", Experimental Thermal and Fluid Science, 40, pp. 126-131, (2012)

[41] Maranzana, G., Perry, I., Maillet, D., "Mini- and micro-channels: influence of axial conduction in the walls," International Journal of Heat and Mass Transfer, 47(17-18), pp. 3993 - 4004, (2004).

[42] Shah, R., Sekulic, D., Fundamentals of Heat Exchanger Design, New York: John Wiley \& Sons, (2003).

[43] Stief, T., Langer, O.U., Schubert, K., "Numerical investigations of optimal heat conductivity in micro heat exchangers," Chemical Engineering Technology, 22(4), pp. 297-303, (1999). 